\title{
Evaluación de la susceptibilidad antifúngica in vitro de T. mentagrophytes y $T$. rubrum
}

\section{In vitro antifungal susceptibility evaluation of T. mentagrophytes and T. rubrum}

\author{
Priscila Plaza-Trujillo $^{1 *}$ (iD, Carmen López-Cisneros ${ }^{1,2}$ iD \\ ${ }^{1}$ Laboratorio de Microbiología Clínica, Facultad de Ciencias Químicas, Universidad de Cuenca, Cuenca, Ecuador. \\ ${ }^{2}$ Facultad de Farmacia, Universidad de Santiago de Compostela, Santiago de Compostela, España. \\ *Autor de correspondencia: priscila.plazat@ucuenca.edu.ec \\ Fecha de recepción: 1 de noviembre de 2021 - Fecha de aceptación: 18 de noviembre de 2021
}

\section{RESUMEN}

El método de dilución en caldo se considera el estándar de oro para la determinación de la concentración inhibitoria mínima (CMI) de antimicrobianos. El objetivo de este trabajo fue la evaluación in vitro de la susceptibilidad antifúngica mediante la estandarización del método de microdilución de T. mentagrophytes y T. rubrum frente a los antifúngicos fluconazol, voriconazol, itraconazol y terbinafina. Para la evaluación de dermatofitos de la colección LMC-FQ de la Sierra Sur del Ecuador se utilizó la metodología de dilución de la guía CLSI M61. Las CIM obtenidas para las cepas de T. mentagrophytes frente a voriconazol son $0.03125 \mu \mathrm{g} / \mathrm{mL}$, frente a itraconazol y terbinafina es $0.00781 \mu \mathrm{g} / \mathrm{mL}$; para T. rubrum se obtuvo un rango de $0.5-4 \mu \mathrm{g} / \mathrm{mL}$ contra fluconazol, $0.01562 \mu \mathrm{g} / \mathrm{mL}$ contra voriconazol y $0.00781 \mu \mathrm{g} / \mathrm{mL}$ contra terbinafina. La estandarización del método se logró replicando la metodología descrita en las guías del CLSI, obteniendo resultados reproducibles y aplicables para un laboratorio de referencia para el diagnóstico micológico. Se pueden reconocer dermatofitos resistentes, y se pueden confirmar, mejorar o modificar las estrategias de tratamiento anti-dermatofitos y contribuir al perfil epidemiológico de la región, así como para pruebas de cribado en la búsqueda de nuevos compuestos naturales o sintéticos con actividad antifúngica.

Palabras clave: Susceptibilidad antifúngica, Trichophyton rubrum, Trichophyton mentagrophytes.

\begin{abstract}
The broth dilution method is considered the gold standard for the Minimum Inhibitory Concentration (MIC) determination of antimicrobials. The aim of this work was the evaluation in vitro of the antifungal susceptibility by standardization of the microdilution method of T. mentagrophytes and T. rubrum against the antifungals fluconazole, voriconazole, itraconazole, and terbinafine. The dilution methodology of the CLSI M61 guideline was used for the evaluation of dermatophytes from the LMC-FQ collection of the South Sierra of Ecuador. The MICs obtained for the strains of T. mentagrophytes against voriconazole are $0.03125 \mu \mathrm{g} / \mathrm{mL}$, against itraconazole and terbinafine is $0.00781 \mu \mathrm{g} / \mathrm{mL}$; for T. rubrum a range of $0.5-4 \mu \mathrm{g} / \mathrm{mL}$ was obtained against fluconazole, $0.01562 \mu \mathrm{g} / \mathrm{mL}$ against voriconazole and $0.00781 \mu \mathrm{g} / \mathrm{mL}$ against terbinafine. Standardization of the method was achieved by replicating the methodology described in the CLSI guidelines, obtaining reproducible and applicable results for a reference laboratory for mycological diagnosis. Resistant dermatophytes can be recognized, and anti-dermatophyte treatment strategies can be confirmed, improved or changed, and contribute to the epidemiological profile of the region, as well as for screening tests in search of new natural or synthetic compounds with antifungal activity.
\end{abstract}

Keywords: Antifungal susceptibility, Trichophyton rubrum, Trichophyton mentagrophytes.

\section{INTRODUCCIÓN}

Los dermatofitos son hongos queratolíticos que afectan la epidermis y anexos cutáneos, produciendo lesiones en las capas superficiales queratinizadas de la piel y faneras como pelos y uñas. Las manifestaciones clínicas pueden ser muy variables, que van desde lesiones superficiales leves, que en forma genérica reciben el nombre de dermatofitosis o tiñas a infecciones crónicas y en casos excepcionales invaden tejidos profundos (Arenas
Guzmán, 2014; Estrada Salazar \& Chacón-Cardona, 2016; Galván-Martínez et al., 2017; Cadavid-Sierra et al., 2013). Las especies más frecuentes aisladas en humanos son $T$. rubrum, T. mentagrophytes y T. tonsurans (Mayorga \& León-Ramírez, 2017; Mazón et al., 1997; Romano, Ghilardi, \& Massai, 2005; Ziegler et al., 2016).

En Latinoamérica las dermatofitosis constituyen una de las infecciones superficiales más prevalentes, con frecuencias de 79.5\% en Venezuela (Capote et al., 2017), 
62.7\% en Brasil (de Albuquerque Maranhão et al., 2019), $52.1 \%$ en Chile (Cruz et al., 2011) y 47\% en Argentina (Davel \& Canteros, 2007). En Ecuador, en estudios de diferentes grupos poblacionales, la prevalencia de las dermatofitosis, según varios autores, se reportan entre el 44 y el $76 \%$, siendo los dermatofitos más frecuentes los del género Trichophyton (Sarango Campoverde, 2015; Campozano \& Heras, 2014; España Gómez \& Espinoza Pizarro, 2019; López Cisneros, Morillo Argudo, \& Plaza Trujillo, 2017).

Hoy en día se observan cepas resistentes a los tratamientos antimicóticos, situación que se da cada vez con mayor frecuencia (Martínez-Rossi et al., 2018), se reporta que la resistencia antifúngica de dermatofitos aislados de pacientes con diferentes manifestaciones clínicas de micosis es alrededor del 19-20\% (Carrillo-Muñoz et al., 2010; Ghannoum, 2016; Manzano-Gayosso et al., 2008). Estos resultados dan relevancia a la aplicación de un método estandardizado en la práctica clínica del diagnóstico micológico. En Ecuador, no existen reportes de estudios de susceptibilidad antifúngica para dermatofitos y no se realizan de forma rutinaria en los laboratorios de diagnóstico, a pesar de que son necesarios para confirmar la existencia de cepas resistentes o el desarrollo de resistencia antifúngica durante el tratamiento en nuestra población, para lo cual, se necesitan métodos para el estudio de sensibilidad que sean reproducibles y disponer de puntos de corte clínicos para la interpretación de sus resultados.

Las pruebas estandarizadas de susceptibilidad antifúngica para dermatofitos, por el método de dilución en caldo considerado gold standard, han sido desarrolladas recientemente por el CLSI (Committee for Clinical and Laboratory Standards), descritas en la guía vigente M61 (2020), basadas en la medición del crecimiento de un inóculo fúngico definido, en presencia de diferentes concentraciones de antifúngicos, permitiendo establecer la concentración mínima inhibitoria (MIC). La distribución del MIC se puede usar para definir poblaciones de hongos de tipo salvaje o no salvaje, información que contribuye al perfil epidemiológico de una población determinando los puntos de corte clínicos y epidemiológicos (CLSI, 2020; Jensen, 2016; Pfaller et al., 2019; Tapia, 2012). El conocimiento sobre la resistencia antifúngica es muy importante y se puede considerar como un primer paso para caracterizar el perfil de resistencia local y con ello optimizar la terapia antifúngica. Los estudios de vigilancia epidemiológica son fundamentales para realizar un seguimiento de las tendencias en resistencia antifúngica y prevenir sus consecuencias, estudios que no existen en el Ecuador (Zurita et al., 2017) y particularmente en la región de la Sierra Sur. El propósito de este trabajo fue evaluar la susceptibilidad antifúngica in vitro de dermatofitos clínicos aislados de la Sierra Sur del Ecuador frente a fluconazol, itraconazol, voriconazol y terbinafina, mediante la estandarización del método de microdilución en caldo para establecer los puntos de corte específicos para las especies en estudio, replicando la metodología de la guía CLSI M61. Los resultados obtenidos de esta estandarización permitirán aplicar este método en el diagnóstico micológico de rutina en los laboratorios para guiar la terapia antifúngica, lo cual servirá para realizar, en futuro, estudios epidemiológicos que incluyan la susceptibilidad a fármacos, caracterización de perfiles de resistencia de dermatofitos aislados de la región y estudios de evaluación de la actividad in vitro de nuevos agentes antifúngicos.

\section{MATERIALES Y MÉTODOS}

El ensayo experimental incluyó seis cepas de dermatofitos de aislados clínicos, T. rubrum $(\mathrm{n}=3), T$. mentagrophytes $(\mathrm{n}=3)$ y dos cepas ATCC como control del ensayo $T$. interdigitale derived from $\mathrm{ATCC} 囚$ 9533 ${ }^{\mathrm{TM} *}$ y T. rubrum derived from ATCC ${ }^{\circledR} 28188^{\mathrm{TM} *}$, de la colección de cepas de hongos del Laboratorio de Microbiología Clínica de la Facultad de Ciencias Químicas (LMC-FQ) de la Universidad de Cuenca, ubicado en la Sierra Sur del Ecuador. Todas las cepas fueron identificadas según sus características macro \& micromorfológicas, pruebas complementarias diagnósticas e identificación molecular.

El método de referencia que se utilizó para la determinación de la susceptibilidad antifúngica fue el de microdilución en caldo documentado en la guía CLSI M61. Los inóculos de las cepas de dermatofitos fueron preparados a partir de colonias puras con 7 días de crecimiento, obteniendo una concentración de $1 \times 10^{3}$ hasta $3 \times 10^{3} \mathrm{UFC} / \mathrm{mL}$ de cada una de las cepas testadas y conservadas a $-70^{\circ} \mathrm{C}$ hasta su uso.

Los antifúngicos testados fueron fluconazol (Bussié), itraconazol (EMS Sigma Pharma), terbinafina (Novartis) y voriconazol (Pfizer). La preparación de la solución seriada de fluconazol fue disuelta en solución salina estéril $0.85 \%$ mientras que terbinafina, itraconazol y voriconazol se disolvieron en dimetilsulfóxido (Sigma-Aldrich). Los rangos de concentración final para fluconazol fueron de $0.125-64 \mu \mathrm{g} / \mathrm{mL}$ y de $0.001-0.5 \mu \mathrm{g} / \mathrm{mL}$ para terbinafina, itraconazol y voriconazol.

El ensayo de susceptibilidad antifúngica se realizó en placas estériles de microtitulación de 96 pocillos, a las cuales se adiciona diferentes concentraciones de antifúngicos en diluciones sucesivas con caldo RPMI 1640 con L-glutamina sin bicarbonato, a pH 7.0 con HEPES (Gibco) e inoculado con la solución stock de esporas. Se incluyó el control de esterilidad (caldo RPMI 1640) y control de crecimiento (caldo RPMI $1640+$ inóculo fúngico). Las placas se incubaron a $30^{\circ} \mathrm{C}$ durante 96 horas o hasta que exista crecimiento visible y posteriormente se realizó la lectura con la técnica de espejo invertido. Todos los ensayos se realizaron por triplicado con 8 repeticiones. Para determinar los puntos de corte del MIC se evaluó el crecimiento en cada pocillo y se comparó con el control de crecimiento y control de esterilidad. Se determinó la media y la moda para cada especie de dermatofito.

\section{RESULTADOS Y DISCUSIÓN}

Se establecieron los valores de MIC para 8 cepas de dermatofitos, 6 cepas de aislados clínicos, distribuidas en T. rubrum $(\mathrm{n}=3), T$. mentagrophytes $(\mathrm{n}=3)$ y 2 cepas control: T. interdigitale derived from ATCC ${ }^{\circledR} 9533^{\mathrm{TM}} * \mathrm{y}$ T. rubrum derived from ATCC $® 28188^{\mathrm{TM}} *$. Los resultados obtenidos fueron comparados con los rangos de MIC y la moda establecidos en la guía CLSI M61. 
Para las cepas de T. rubrum de aislados clínicos, se obtuvo un rango de MIC de $2-1 \mu \mathrm{g} / \mathrm{mL}$ para fluconazol; todas las cepas testadas, incluida la cepa control, estuvieron dentro del rango de referencia de $0.5-4 \mu \mathrm{g} / \mathrm{mL}$ y cerca de la moda con un valor de $1 \mu \mathrm{g} / \mathrm{mL}$. Para voriconazol el MIC fue $0.01562 \mu \mathrm{g} / \mathrm{mL}$, valor dentro del rango de la guía M61. El MIC para terbinafina fue de $0.00781 \mu \mathrm{g} / \mathrm{mL}$ (Tabla 1 , Fig. 1).

Para T. mentagrophytes de aislados clínicos el MIC frente a voriconazol fue de $0.03125 \mu \mathrm{g} / \mathrm{mL}$, para itraconazol y terbinafina el MIC fue de $0.00781 \mu \mathrm{g} / \mathrm{mL}$ (Fig. 2), los puntos de corte para voriconazol y terbinafina se encuentran dentro del rango reportado en la guía M61 y los valores de MIC son cercanos a la moda $(0.06 \mu \mathrm{g} / \mathrm{mL})$. La cepa control $T$. interdigitale derived from ATCC 9533 ${ }^{\mathrm{TM}} *$ para voriconazol tuvo un MIC de $0.0625 \mu \mathrm{g} / \mathrm{mL}$ y para itraconazol de $0.03125 \mu \mathrm{g} / \mathrm{mL}$, rangos que están dentro de la guía de referencia. Para terbinafina, el valor de MIC fue de $0.03125 \mu \mathrm{g} / \mathrm{mL}$ (Tabla 2).

Este es el primer reporte sobre la estandarización del método de susceptibilidad antifúngica para dermatofitos en la región de la Sierra Sur del Ecuador. Los resultados de esta investigación generan un precedente del perfil de susceptibilidad antifúngica de dermatofitos, que permitirán definir o refinar los puntos de corte o los valores de corte epidemiológico en la región. Es importante indicar que los perfiles de susceptibilidad antifúngica no son los mismos en todas las regiones, por lo que se debe impulsar este campo de estudio (Jarabrán et al., 2015; Pérez-Cárdenas, Hoyos Zuluaga, \& Henao, 2013; da Silva Barros, de Assis Santos, \& Soares Hamdan, 2007; Berkow, Lockhart, \& Ostrosky-Zeichner, 2020). En el método de susceptibilidad antifúngica por microdilución en caldo, los parámetros como el medio de cultivo, tamaño del inóculo, tiempo de incubación, entre otros, influyen directamente en los resultados, por lo que se consideran puntos críticos dentro de la metodología, es importante cumplir con todas las condiciones experimentales descritas en la guía CLSI M61 (Takasuka, 2000; Fohrer et al., 2006; Castro Méndez, García Sánchez, \& Martín-Mazuelos, 2019; Dogra, Shaw, \& Rudramurthy, 2019; Poojary et al., 2019).

Varios autores han reportado los puntos de corte para la susceptibilidad antifúngica de dermatofitos utilizando la guía CLSI. Las cepas testadas para fluconazol y voriconazol en este estudio presentaron valores de MIC cercanos y dentro de los rangos y la moda establecidos en la guía de referencia (CLSI, 2020), concordando con otros ensayos realizados en Latinoamérica con cepas de aislamientos clínicos (Jarabrán et al., 2015; da Silva Barros et al., 2007); así como se difiere con el reporte de Santos et al. (2010), realizado en Argentina, que presenta valores más altos de MIC.

Según Maurya et al. (2019) y Bhatia \& Sharma (2015), en los estudios realizados con dermatofitos aislados de muestras clínicas de pacientes de la India, muestran MICs por debajo del rango establecido en la M61 frente a itraconazol. En el presente estudio, para las cepas de $T$. mentagrophytes de aislados clínicos, se obtuvieron valores de MIC $(0.00781 \mu \mathrm{g} / \mathrm{mL})$ para itraconazol, más bajos que el rango y la moda establecidos en la guía, concordando con lo reportado en un estudio en Colombia, que establece el rango entre $0.0009-4 \mu \mathrm{g} / \mathrm{mL}$ en 13 cepas aisladas de muestras clínicas (Pérez-Cárdenas et al., 2013). Este resultado se puede atribuir a los aspectos

Tabla 1. Rangos de MIC de T. rubrum frente a antifúngicos por el método de microdilución en caldo.

\begin{tabular}{|c|c|c|c|c|c|}
\hline \multirow{3}{*}{ Specie } & \multirow{2}{*}{\multicolumn{2}{|c|}{$\begin{array}{c}\text { Fluconazol } \\
\text { MIC }(\mu \mathrm{g} / \mathrm{mL})\end{array}$}} & \multirow{2}{*}{\multicolumn{2}{|c|}{$\begin{array}{c}\text { Voriconazol } \\
\text { MIC }(\mu \mathrm{g} / \mathrm{mL})\end{array}$}} & \multirow{3}{*}{$\begin{array}{l}\text { Terbinafina } \\
\text { MIC }(\mu \mathrm{g} / \mathrm{mL}) \\
\text { LMC-FQ }\end{array}$} \\
\hline & & & & & \\
\hline & LMC-FQ & CLSI-M61 & LMC-FQ & CLSI-M61 & \\
\hline $\begin{array}{l}\text { T. rubrum cepas clínicas } \\
(\mathrm{n}=3)\end{array}$ & $1.0-4.0$ & $0.5-4.0$ & 0.01562 & $0.008-0.06$ & 0.00781 \\
\hline $\begin{array}{l}\text { T. rubrum derived from } \\
\text { ATCC } ® 28188^{\mathrm{TM} *}(\mathrm{n}=1)\end{array}$ & $1.0-2.0$ & $0.5-4.0$ & $\begin{array}{r}0.00781- \\
0.01562\end{array}$ & $0.008-0.06$ & 0.00781 \\
\hline
\end{tabular}

LMC-FQ: Laboratorio de Microbiología Clínica de la Facultad de Ciencias Químicas de la Universidad de Cuenca.

CLSI-M61: Performance Standards for Antifungal Susceptibility Testing of Filamentous Fungi 2nd Edition.

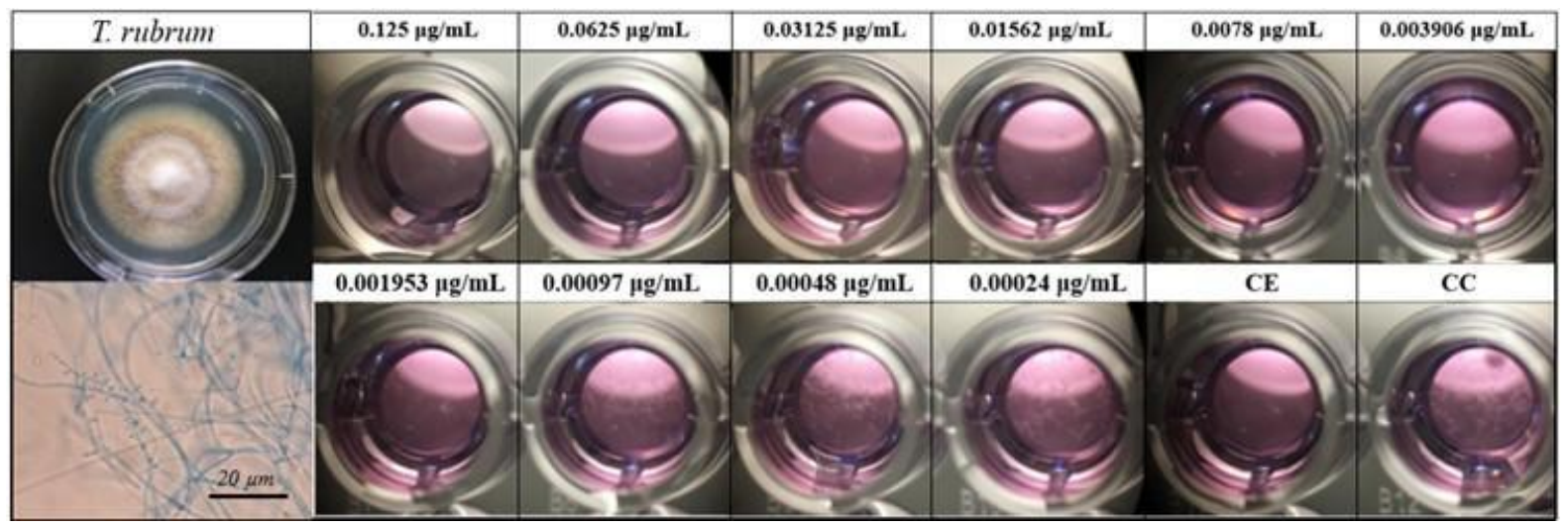

Figura 1. T. rubrum frente a terbinafina. Colonia en medio de SDA, microfotografía de estructuras fúngicas con azul de lactofenol (40X). Microfotografía de pocillos con concentraciones decrecientes de antifúngico, de 0.125 a $0.00024 \mu \mathrm{g} / \mathrm{mL}$. $\mathrm{MIC}=0.0078 \mu \mathrm{g} / \mathrm{mL}$. CE=columna de control de esterilidad, $\mathrm{CC}=$ columna de control de crecimiento (10X). 
Tabla 2. Rangos de MIC de T. mentagrophytes frente a antifúngicos por el método de microdilución en caldo.

\begin{tabular}{lccccc}
\hline \multirow{2}{*}{ Specie } & \multicolumn{2}{c}{ Voriconazol } & \multicolumn{2}{c}{ Itraconazol } & Terbinafina \\
\cline { 2 - 5 } & \multicolumn{2}{c}{ MIC $(\mu \mathrm{g} / \mathrm{mL})$} & LMC $(\mu \mathrm{g} / \mathrm{mL})$ & MIC $(\mu \mathrm{g} / \mathrm{mL})$ \\
\cline { 2 - 5 } & LMC-FQ & CLSI-M61 & CLSI-M61 & LMC-FQ \\
\hline $\begin{array}{l}\text { T. mentagrophytes cepas } \\
\text { clínicas (n=3) }\end{array}$ & 0.03125 & $0.03-0.25$ & 0.00781 & $0.03-0.25$ & 0.00781 \\
\hline $\begin{array}{l}\text { Trichophyton inter-digital } \\
\text { derived from ATCC } \\
\text { 9533 }\end{array}$ & 0.0625 & $0.03-0.25$ & 0.03125 & $0.03-0.25$ & 0.03125 \\
\hline
\end{tabular}

LMC-FQ: Laboratorio de Microbiología Clínica de la Facultad de Ciencias Químicas de la Universidad de Cuenca.

CLSI-M61: Performance Standards for Antifungal Susceptibility Testing of Filamentous Fungi 2nd Edition.

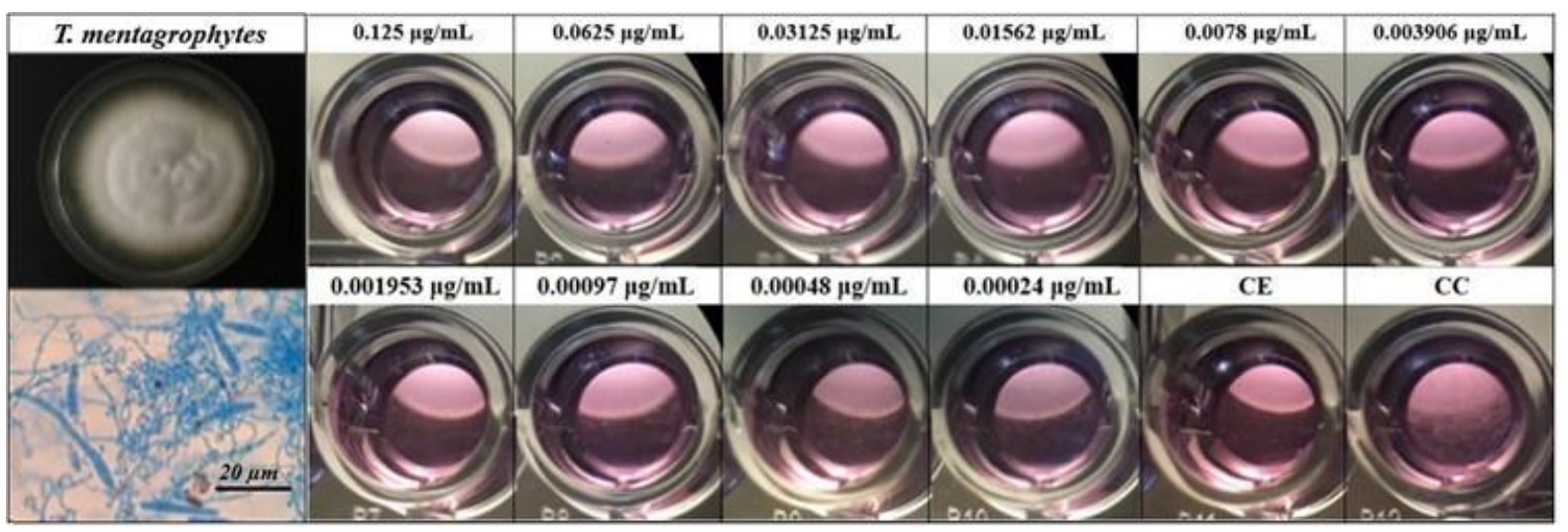

Figura 2. T. mentagrophytes frente a itraconazol. Colonia en medio de SDA, microfotografía de estructuras fúngicas con azul de lactofenol (40X) (López Cisneros et al., 2021). Microfotografía de pocillos con concentraciones decrecientes de antifúngico, de 0.125 a $0.00024 \mu \mathrm{g} / \mathrm{mL}$. MIC=0.0078 $\mu \mathrm{g} / \mathrm{mL}$. CE=columna de control de esterilidad, $\mathrm{CC}=$ columna de control de crecimiento (10X).

clínicos del paciente, como por ejemplo el tipo de lesión y la no exposición a este agente antifúngico, lo que puede ser un aspecto que influya en los resultados de susceptibilidad (Castro Méndez et al., 2019).

Estudios realizados con cepas de dermatofitos aislados en Chile reportan rangos de MIC para terbinafina de 0.03$0.06 \mu \mathrm{g} / \mathrm{mL}$ frente a $T$. mentagrophytes, mostrando mayor sensibilidad que el rango de la guía CLSI, concordando con lo reportado en Colombia y con los valores obtenidos en el presente estudio (Carillo-Muñoz et al., 2008, 2013; Carillo-Muñoz et al., 2010; Jarabrán et al., 2015; Diaz et al., 2002; Espinel-Ingroff et al., 2007; Pérez-Cárdenas et $a l ., 2013)$. En el caso de la terbinafina para T. interdigitale derived from ATCC ${ }^{\circledR} 9533^{\mathrm{TM}}$ *, el valor de MIC es similar a los estudios realizados en India por Adimi et al. (2013), mientras que para $T$. rubrum el MIC de terbinafina concuerda con los resultados obtenidos en Brasil por da Silva Barros et al. (2007). Cabe destacar que el punto de corte obtenido para terbinafina frente a $T$. rubrum no se encuentra descrito en la guía CLSI, a pesar de su amplio uso terapéutico en tinea pedis, por lo que, este es un resultado muy importante en la determinación de susceptibilidad antifúngica.

La detección de resistencia a los medicamentos antifúngicos permite cambiar o ajustar las terapias de acuerdo con la sensibilidad y conocer la existencia de cepas resistentes en la región. A pesar de la importancia de los estudios de susceptibilidad, en nuestro medio, estos métodos no están siempre disponibles y no hay estudios epidemiológicos que evalúen la susceptibilidad a los antimicóticos disponibles. Varios autores recomiendan contar con programas de vigilancia epidemiológica fúngica, con lo que se apoyaría a la realización de estas pruebas y se conocería las tasas de resistencia antifúngica (Berkow et al., 2020; Quindós, 2018; Tapia, 2012; Zapata \& Cardona, 2012).

\section{CONCLUSIONES}

La estandarización del método se logró mediante la replicación de la metodología descrita en la guía CLSI M61, obteniéndose resultados reproducibles y aplicables para un laboratorio de referencia de diagnóstico micológico. La aplicación del método de microdilución en caldo in vitro para dermatofitos en nuestro medio permitirá identificar cepas resistentes para confirmar, mejorar o cambiar las estrategias de tratamiento antidermatofítico y contribuir al perfil epidemiológico de la región. Además, es de gran utilidad para ensayos de screening para la búsqueda de nuevos compuestos naturales o sintéticos con actividad antimicótica.

\section{AGRADECIMIENTO}

Agradecemos a la Facultad de Ciencias Químicas de la Universidad de Cuenca y al Departamento de Química Orgánica, Facultade de Farmacia de la Universidad Santiago de Compostela. 


\section{REFERENCIAS}

Adimi, P., Jamal Hashemi, S., Mahmoudi, M., Mirhendi, H., Reza Shidfar, M., Emmami, M., RezaeiMatehkolaei, A., Gramishoar, M., \& Kordbacheh, P. (2013). In-vitro activity of 10 antifungal agents against 320 dermatophyte strains using microdilution method in Tehran. Iranian Journal Pharmaceutical Research, 12(3), 537-45.

Arenas Guzmán, R. (2014). Micología medica ilustrada. McGraw-Hill Interamericana.

Berkow, E. L., Lockhart, S. L., \& Ostrosky-Zeichner, L. (2020). Antifungal susceptibility testing: Current approaches. Clinical Microbiology Reviews, 33(3), e00069-19. Doi:10.1128/CMR.00069-19

Bhatia, V. K., \& Sharma, P. C. (2015). Determination of minimum inhibitory concentrations of itraconazole, Terbinafine and Ketoconazole against dermatophyte by broth microdilution method. Indian Journal of Medical Microbiology, 33(4):533-37. doi:10.4103/02550857.167341

Cadavid Sierra, M., Santa, C., Colmenares, L. M., Velez, L. M., Mejía, M. A., Restrepo Jaramillo, B. N., \& Cardona Castro, N. M. (2013). Estudio etiológico y epidemiológico de las micosis cutáneas en un laboratorio de referencia, Antioquia, Colombia. CES Medicina, 27(1), 7-20. doi:10.21615/ces med.v27i1.2495

Campozano, N., \& Heras, V. (2014). Determinación de la prevalencia de dermatofitosis en los niños de la escuela de educación general básica Padre Juan Bautista Aguirre de La Parroquia Miraflores de la ciudad de Cuenca. Universidad de Cuenca, Tesis de pregrado, 87 págs.

http://dspace.ucuenca.edu.ec/handle/123456789/21010

Capote, A. M., Ferrara, G., Mercedes Panizo, M., García, N., Alarcón, V., \& Dolande, M. (2017). Utilidad del Litmus Milk® para la diferenciación de los complejos de especies Trichophyton rubrum y Trichophyton mentagrophytes. Revista de la Sociedad Venezolana de Historia de la Medicina, 37(2), 78-81.

Carrillo-Muñoz, A-J., Tur-Tur, C., Cárdenes, D., Rojas, F., \& Giusiano, G. (2013). Influence of the ecological group on the in-vitro antifungal susceptibility of dermatophytic fungi. Revista Iberoamericana de Micología, 30(2), 130-33. doi:10.1016/j.riam.2012.12.002

Carrillo-Muñoz, A-J., Tur-Tur, C., Hernández-Molina, JM., Santos, P., Cárdenes, D., \& Giusiano, G. (2010). Antifúngicos disponibles para el tratamiento de las micosis ungueales. Revista Iberoamericana de Micología, 27(2), 49-56. doi:10.1016/j.riam.2010.01.007

Carrillo-Muñoz, A-J., Giusiano, G., Cárdenes, D., Hernández-Molina, J-M., Eraso, E., Quindós, G., Guardia, C., del Valle, O., Tur-Tur, C., \& Guarro, J. (2008). Terbinafine susceptibility patterns for Onychomycosis-Causative and Scopulariopsis Brevicaulis. International Journal of Antimicrobial Agents, 31(6), 540-43. doi:10.1016/j.ijantimicag.2008.01.023

Castro Méndez, C., García Sánchez, E., \& MartínMazuelos, E. (2019). Actualización de los métodos de estudio de sensibilidad in vitro a los antifúngicos. Enfermedades Infecciosas y Microbiología Clínica, 37(S1), 32-39. doi:10.1016/S0213-005X(19)30180-6

CLSI. (2020). Performance standards for antifungal susceptibility testing of Filamentous Fungi (2nd ed.). CLSI supplement M61. Wayne, PA: Clinical and Laboratory Standards Institute Wayne.

Cruz, R., Ponce, E., Calderón, L., Delgado, N., Vieille, P., \& Piontelli, E. (2011). Superficial mycoses in the city of Valparaiso, Chile: Periodo 2007-2009. Revista Chilena Infectología, 28(5), 404-409.

Davel, G., \& Canteros, C. E. (2007). Epidemiological status of mycoses in the Argentine Republic. Revista Argentina de Microbiología, 39(1), 28-33.

de Albuquerque Maranhão, F. C., Oliveira-Júnior, J. B., Dos Santos Araújo, M. A., \& Wanderlei Silva, D. M. (2019). Mycoses in Northeastern Brazil: Epidemiology and prevalence of fungal species in 8 years of retrospective analysis in Alagoas. Brazilian Journal of Microbiology, 50(4), 969-78. doi:10.1007/s42770-01900096-0

Diaz, M. C., Roessler, P., Fich, F., Gómez, O., Ostornol, P., \& Pérez, L. (2002). Dermatofitosis. etiología y susceptibilidad antifúngica in vitro en tres centros hospitalarios de Santiago (Chile). Boletín Micológico, 17, 101-107. doi:10.22370/bolmicol.2002.17.0.445

Dogra, S., Shaw, D., \& Rudramurthy, S. M. (2019). Antifungal drug susceptibility testing of dermatophytes: Laboratory findings to clinical implications. Indian Dermatology Online Journal, 10(3), 225-233. doi:10.4103/idoj.IDOJ_146_19

España Gómez, S. E., \& Espinoza Pizarro, T. M. (2019). Situación de la micosis superficial en Ecuador. Universidad Católica de Santiago de Guayaquil, Facultad de Ciencias Médicas, Trabajo de Titulación Carrera de Enfermería. Disponible en http://repositorio.ucsg.edu.ec/handle/3317/12568

Espinel-Ingroff, A., Fothergill, A., Ghannoum, M., Manavathu, E., Ostrosky-Zeichner, L., Pfaller, M. A., Rinaldi, M. G., Schell, W., \& Walsh, T. J. (2007). Quality control and reference guidelines for CLSI broth microdilution method (M38-A Document) for susceptibility testing of anidulafungin against molds. Journal of Clinical Microbiology, 45(7), 2180-2182. doi:10.1128/JCM.00399-07

Estrada Salazar, G. I., \& Chacón-Cardona, J. A. (2016). Frecuencia de dermatomicosis y factores asociados en población vulnerable de la ciudad de Manizales. Colombia. 2011. Revista de Salud Pública, 18(6), 953. doi:10.15446/rsap.v18n6.51794

Fohrer, C., Fornecker, L., Nivoix, Y., Cornila, C., Marinescu, C., \& Herbrecht, R. (2006). Antifungal combination treatment: a future perspective. International Journal of Antimicrobial Agents, 27(Suppl 1), 25-30.

doi:10.1016/j.ijantimicag.2006.03.016

Galván-Martínez, I. L., Fernández-Martínez, R., NarroLlorente, R., Moreno Coutiño, G., \& Arenas, R. (2017). Frecuencia de tiña del cuerpo en un hospital del Estado de Quintana Roo. Medicina Interna de México, 33(1), 5-11.

Ghannoum, M. (2016). Azole resistance in dermatophytes: Prevalence and mechanism of action. 
Journal of the American Podiatric Medical Association, 106(1), 79-86. Doi:10.7547/14-109

Jarabrán, M. C. D., Pablo Díaz González, M. C., Rodríguez, J. E., \& Carrillo Muñoz, A. J. (2015). Evaluación del perfil de sensibilidad in vitro de aislamientos clínicos de Trichophyton mentagrophytes y Trichophyton rubrum en Santiago, Chile. Revista Iberoamericana de Micología, 32(2), 83-87. doi:10.1016/j.riam.2013.12.002

Jensen, R. H. (2016). Resistance in human pathogenic yeasts and filamentous fungi: Prevalence, underlying molecular mechanisms and link to the use of antifungals in humans and the environment. Danish Medical Journal, 63(10), B5288.

López Cisneros, C. L., Cazar Ramírez, M. E., BailonMoscoso, N., Guardado, E., Borges, F., Uriarte, E., \& João Matos, M. (2021). Study of a selected series of 3- and 4-arylcoumarins as antifungal agents against dermatophytic fungi: $\mathrm{T}$. rubrum and $\mathrm{T}$. mentagrophytes. ChemistrySelect, 6(37), 9981-89. doi:10.1002/slct.202103099

López Cisneros, C. L., Morillo Argudo, D. A., \& Plaza Trujillo, P. L. (2017). Estudio trasversal: Micosis superficiales en niños escolares de una parroquia rural de Cuenca, Ecuador. Revista Médica Hospital Del José Carrasco Arteaga, 9(3), 249-54. doi:10.14410/2017.9.3.ao.41

Manzano-Gayosso, P., Méndez-Tovar, L. J., HernándezHernández, F., \& López-Martínez, R. (2008). La resistencia a los antifúngicos: Un problema emergente en México. Gaceta Médica de México, 144(1), 23-26.

Martínez-Rossi, N. M., Bitencourt, T. A., Peres, N. T. A., Lang, E. A. S., Gomes, E. V., Quaresemin, N. R., Martins, M. P., Lopes, L., \& Rossi, A. (2018). Dermatophyte resistance to antifungal drugs: Mechanisms and prospectus. Front Microbiology, 9, 1108. doi:10.3389/fmicb.2018.01108

Maurya, V. K., Kachhwaha, D., Bora, A., Khatri, P. K., \& Rathore, L. (2019). Determination of antifungal minimum inhibitory concentration and its clinical correlation among treatment failure cases of dermatophytosis. Journal Family Medicine Primary Care, 8(8), 2577-81. doi:10.4103/jfmpc.jfmpc_483_19

Mayorga, J., \& de León-Ramírez, R. M. (2017)

Prevalencia de dermatofitosis producidas por Trichophyton rubrum. Dermatología Revista Mexicana, 61(2), 108-114.

Mazón, A., Salvo, S., Vives, R., Valcayo, A., \& Sabalza. M. A. (1997). Etiologic and epidemiologic study of dermatomycoses in Navarra (Spain). Revista Iberoamericana de Micología, 14(2), 65-68.

Pérez-Cárdenas, J. E., Hoyos Zuluaga, A. M., \& Cárdenas Henao, C. (2013). Sensibilidad antimicótica de diferentes especies de hongos de pacientes con micosis ungueal en la ciudad de Manizales (Caldas, Colombia). Biosalud, 11(2), 26-39.

Pfaller, M. A., Huband, M. D., Flamm, R. K., Bien, P. A. \& Castanheira, M. (2019). In vitro activity of
APX001A (Manogepix) and comparator agents against 1,706 fungal isolates collected during an International Program in 2017. Antimicrobial Agents and Chemotherapy, 63(8), 1-11. doi:10.1128/AAC.0084019

Poojary, S., Miskeen, A., Bagadia, J., Jaiswal, S., \& Uppuluri, P. (2019). A study of in vitro antifungal susceptibility patterns of dermatophytic fungi at a tertiary care center in Western India. Indian Journal of Dermatology, 64(4), 277-84.

Quindós, G. (2018). Epidemiología de las micosis invasoras: Un paisaje en continuo cambio. Revista Iberoamericana de Micología, 35(4), 171-78. doi:10.1016/j.riam.2018.07.002

Romano, C., Ghilardi, A., \& Massai, L. (2005). Eightyfour consecutive cases of Tinea Faciei in Siena, a retrospective study (1989-2003). Mycoses, 48(5), 343-346. doi:10.1111/j.1439-0507.2005.01138.x

Santos, P. E., Córdoba, S., Rodero, L. L., CarrilloMuñoz, A. J., \& Lopardo, H. A. (2010). Tinea capitis. Experiencia de 2 años en un hospital de pediatría de Buenos Aires, Argentina. Revista Iberoamericana de Micología, 27(2), 104-106. doi:10.1016/j.riam.2010.01.004

Sarango Campoverde, N. O. (2015). Agentes causales de micosis superficiales en pacientes que acuden al Laboratorio Biolab del cantón Yantzaza. Universidad Nacional de Loja, Carrera de Laboratorio Clínico: Trabajo de Titulación.

http://dspace.unl.edu.ec/jspui/handle/123456789/13651

da Silva Barros, M. E., de Assis Santos, D., Soares Hamdan, J. (2007). Evaluation of susceptibility of Trichophyton mentagrophytes and Trichophyton rubrum clinical isolates to antifungal drugs using a modified CLSI microdilution method (M38-A). Journal of Medical Microbiology, 56, 514-518. doi:10.1099/jmm.0.46542-0

Takasuka, T. (2000). Amino acid- or protein-dependent growth of Trichophyton mentagrophytes and Trichophyton rubrum. FEMS Immunology and Medical Microbiology, 29(4), 241-45. doi:10.1111/j.1574695X.2000.tb01529.x

Tapia, C. (2012). Antifúngicos y resistencia. Revista Chilena de Infectología, 29(3), 357-357. doi:10.4067/S0716-10182012000300020

Zapata, F., \& Cardona, N. (2012). What we must know about antifungal susceptibility testing. CES Medicina, 26(1), 71-83.

Ziegler, W., Lempert, S., Goebeler, M., \& Kolb-Mäurer, A. (2016). Tinea capitis: temporal shift in pathogens and epidemiology. Journal Der Deutschen Dermatologischen Gesellschaft, 14(8), 818-25. doi:10.1111/ddg. 12885

Zurita, J., Denning, D. W., Paz-Y-Miño, A., Solís, M. B., \& Arias, L. M. (2017). Serious fungal infections in Ecuador. European Journal of Clinical Microbiology \& Infectious Diseases, 36(6), 975-981. doi:10.1007/s10096-017-2928-5 